\title{
Rapid and repeated accretion of planetesimals in an outer region of the protoplanetary disc
}

\author{
NING MA ${ }^{1}$, WLADIMIR NEUMANN ${ }^{2}$, ADRIEN NÉRI $^{1}$, \\ WINFRIED H. SCHWARZ ${ }^{2}$, THOMAS LUDWIG ${ }^{2}$, MARIO \\ TRIELOFF $^{2}$, HUBERT KLAHR ${ }^{3}$ AND AUDREY BOUVIER ${ }^{1}$ \\ ${ }^{1}$ Universität Bayreuth \\ ${ }^{2}$ Universität Heidelberg \\ ${ }^{3}$ Max-Planck-Institut für Astronomie \\ Presenting Author: audrey.bouvier@uni-bayreuth.de
}

Isotopic anomalies in meteorites have revealed two reservoirs of planetesimal formation corresponding to non-carbonaceous (NC) and carbonaceous (C) compositions sampling the inner and more outer regions of the protoplanetary disc, respectively [1], or possibly formed at different periods [2]. Chronological studies provide constraints on the timing of such separation lasting between 1 and 4 Myr after CAIs. Generally, differentiated planetesimals are found to be prevalent in the $\mathrm{NC}$ region and accreted within $<0.5-2 \mathrm{Myr}$ after solar system formation, forming before undifferentiated $\mathrm{NC}$ and $\mathrm{C}$ parent bodies (1.5 to $4 \mathrm{Myr}$ ). $\mathrm{NC}$ magmatic iron parent bodies (PB) started accreting slightly before those of $\mathrm{C}$ irons but all within $1 \mathrm{Myr}$ [1].

We identify a first group of partially differentiated primitive achondrites which are isotopically related to the CR2 chondrites. We propose the name of Tafassites after the Tafassasset meteorite. These meteorites are currently classed as CR6-7, ungrouped chondrites and achondrites, or primitive achondrites. Their mineralogical assemblage, equilibration temperature, $f \mathrm{O}_{2}$, and U-Pb SIMS chronological records in merrillites provide constraints on the formation and thermal evolution of the Tafassite PB (TPB). Based on ${ }^{17} \mathrm{O}-{ }^{54} \mathrm{Cr}^{5}{ }^{50} \mathrm{Ti}$ isotopic anomalies [3], Tafassites are distinct from CR2 chondrite and NWA 6704 differentiated (ungrouped) achondrite PBs. Although Tafassites are isotopically similar to the NWA 011 differentiated (ungrouped) achondrite, they do not share the same PB due to their geochemical compositions. Numerical modelling best fit TPB accretion at $1.1 \mathrm{Myr}$ after CAIs with a corresponding radius of 100-200 km. The TPB formed before the CR-related NWA 011 and NWA $6704 \mathrm{PBs} \sim 1.5 \mathrm{Myr}$ [3], and CR2 chondrite PB 3-4Myr [1].

Our results indicate that the TPB formed concurrently with NC primitive achondrite $\mathrm{PB}$ [4]. The similar isotopic anomalies, particularly for ${ }^{54} \mathrm{Cr}$, of CR-related planetesimals suggest that limited radial mixing of carrier grains occurred throughout the period of their parent body accretion (from 1 to $4 \mathrm{Myr}$ after CAIs) within this particular region of the protoplanetary disc.

[1] Kruijer et al. (2020), Nature Astronomy 4, 32-40.

[2] Lichtenberg et al. (2021), Science 371, 365-370.

[3] Sanborn et al. (2019), GCA 245, 577-596.

[4] Neumann et al. (2018), Icarus 311, 146-169. 